\title{
Steroids Significantly Decrease Postoperative Postural Hypotension in Total Knee Replacement
}

\author{
Gloria H. M. Cheng, MBBS, MRCS(Ire) ${ }^{1}$ Gabriel K. Y. Tan ${ }^{2} \quad$ Muhammad F. B. Kamarudin, MBBS $^{1}$ \\ Bryan W. W. Lee, MBBS ${ }^{1}$ Yap Yan Mei, MSc, BSc (Hon) ${ }^{3}$ Kelvin G. Tan, MBBS, FRCS(Edin) ${ }^{1}$
}

${ }^{1}$ Department of Orthopaedic Surgery, Tan Tock Seng Hospital, Singapore, Singapore

${ }^{2}$ Lee Kong Chian School of Medicine, Nanyang Technological University, Singapore

${ }^{3}$ Department of Physiotherapy, Tan Tock Seng Hospital, Singapore

J Knee Surg 2023;36:208-215.
Address for correspondence Kelvin G. Tan, Department of Orthopaedic Surgery, 11 Jalan Tan Tock Seng Hospital, Singapore 308433 (e-mail: kelvin_tan@ttsh.com.sg).

\begin{abstract}
Keywords

- total knee replacement

- dexamethasone

- postural hypotension

Total knee replacement (TKR) is one of the most common orthopaedic procedures performed, and enhanced recovery after surgery (ERAS) has been developed and incorporated into inpatient surgical pathways to improve patient outcomes. Under ERAS recommendations, multimodal prophylaxis has been used to help manage postoperative nausea and vomiting (PONV) following TKR. Dexamethasone is one of the commonly used for this and the anti-inflammatory properties could depress vagal activity, reducing postural hypotension $(\mathrm{PH})$. The hypothesis of this study is that postoperative dexamethasone use is associated with lower rates of early postoperative PH following TKR surgery. In our institution, patients who undergo elective primary TKR are admitted on the day of surgery and follow a standardized ERAS protocol. Data on patients who underwent elective primary TKR under a single adult reconstruction team from September 2017 to March 2020 were reviewed and analyzed. A review of demographic characteristics, surgical data, postoperative medications, and postoperative notes was performed. Binary logistic regression was used to assess the effect of the use of dexamethasone on $\mathrm{PH}$, with an adjusted odds ratio (OR) calculated after accounting for potential confounders. Of the 149 patients were included in the study, 78 had dexamethasone postoperatively, and 71 did not. Patients who had received dexamethasone were statistically less likely to suffer from $\mathrm{PH}(\mathrm{OR}=0.31, p=0.03)$ and less likely to develop PONV $(O R=0.21, p=0.006)$. Patients who had received dexamethasone were more likely able to participate in early physiotherapy $(O R=2.42$, $p=0.14$ ), and this result was statistically insignificant. The use of postoperative intravenous dexamethasone is significantly associated with lower rates of postoperative PH after TKR. However, more studies are required to assess the optimal dosing amount and frequency, as well as to assess other factors which can enhance early postoperative patient mobilization as part of our goals for ERAS. This therapeutic study reflects level of evidence III.
\end{abstract}

received

February 8, 2021

accepted after revision

May 31, 2021

article published online

July 8, 2021 (c) 2021. Thieme. All rights reserved.

Thieme Medical Publishers, Inc.,

333 Seventh Avenue, 18th Floor,

New York, NY 10001, USA
DOI https://doi.org/

10.1055/s-0041-1731722. ISSN 1538-8506. 
Total knee replacement (TKR) is one of the most common orthopaedic procedures performed, and enhanced recovery after surgery (ERAS) protocols have been incorporated into various inpatient surgical pathways to reduce length of stay, reduce postoperative complications, and improve patient outcomes. Under ERAS recommendations, multimodal prophylaxis for postoperative nausea and vomiting (PONV) include dopamine antagonists, serotonin antagonists, and corticosteroids. ${ }^{1}$

Surgery causes an inflammatory state, and the postoperative systemic inflammatory response syndrome (SIRS) triggers parasympathetic or vagal activity, also known as the "inflammatory reflex" which could result in postural hypotension $(\mathrm{PH}){ }^{2,3}$

Dexamethasone is commonly given perioperatively to reduce $\mathrm{PONV},{ }^{4}$ and we postulate that its anti-inflammatory properties could depress vagal activity and hence reduce the rate of $\mathrm{PH}$ in the postoperative patients. ${ }^{5}$ By doing so, this will allow for successful early mobilization, quicker return to function, and decreased postoperative morbidity and complications. Our hypothesis is that postoperative dexamethasone use is associated with lower rates of early postoperative PH following TKR surgery.

We aim to assess the prevalence of postoperative $\mathrm{PH}$ and the effects of dexamethasone on rates of PH. Secondary aims are to investigate the effects of dexamethasone on PONV, and on patient's ability to ambulate on postoperative day (POD) and participate in physiotherapy activities.

\section{Materials/Patients and Methods}

This is an ethics board approved study.

This is a retrospective cohort study, involving patients who underwent elective primary TKR under a single adult reconstruction team in Tan Tock Seng Hospital, Singapore. Data from patients who underwent surgery between September 2017 and March 2020 were retrospectively reviewed and analyzed. Patients with missing data were excluded.

\section{Total Knee Replacement Enhanced Recovery after Surgery Protocol in Tan Tock Seng Hospital}

In our institution, patients who undergo elective primary TKR are admitted on the day of surgery and follow a standardized ERAS protocol. ${ }^{6}$

\section{Preoperative Phase}

Patients receive preoperative information, education, and counseling with the surgeon and physiotherapist after the timing of surgery is set. About 2 weeks prior to surgery, they are reviewed by the anesthetist for preadmission assessment and optimization.

\section{Standard Anesthetic Protocol}

Either general anesthesia or spinal anesthesia is administered based on evaluation and recommendation by the anesthetist and decision of the patient, with spinal anesthesia being the preferred option. Intraoperatively, normothermia is maintained with the use of air warmers, and prevention of perioperative blood loss is done through the routine use of tranexamic acid. Intravenous antibiotics (usually cefazolin) for antimicrobial prophylaxis is administered after induction, prior to skin incision.

\section{Perioperative Surgical Factors}

A thigh tourniquet is routinely used to reduce bleeding, especially during cement insertion, barring any contraindications to its use, that is, peripheral arterial disease in the lower limb. Local infiltrative analgesia comprising ketorolac, ropivacaine $1 \%$, normal saline, and adrenaline $(1: 1,000)$ is administered into the knee joint capsule, ligaments, and quadriceps.

\section{Postoperative Phase}

The patient is transferred to a specialized adult reconstruction postoperative general ward. A standardized medication regime for postoperative analgesia and prophylaxis for PONV is started. This consists of regular paracetamol, cyclooxygenase-2 inhibitors, such as celecoxib and gabapentin, and oxycodone with naloxone controlled release tablets, intravenous ondansetron $4 \mathrm{mg}$ in every 8 hours for 24 hours, and intravenous dexamethasone 4 to $8 \mathrm{mg}$ in every 8 hours for 24 hours. The reason for the 24-hour dosing is because of the high incidence of PONV during this period. ${ }^{7}$ Additionally, regional block of the operated knee or lower limb or patient controlled analgesia may be administered by the anesthetist if the pain control is suboptimal with above analgesia. To maintain balanced fluid management, an intravenous fluid drip of normal saline $1 \mathrm{~L}$ over 24 hours is given.

Serum hemoglobin level is checked in the morning of the first POD, and patient is transfused accordingly if indicated. To achieve early mobilization, the patient is started on a welldefined, fast-track rehabilitation regime by the physiotherapists. Physiotherapy sessions started on the day of surgery or on POD 1 if the patient reaches the ward beyond 5 p.m. Standardized recording of blood pressure is performed in supine position and next in standing position. Therapy is discontinued if patient has $\mathrm{PH}$, is unable to tolerate mobilization, or is symptomatic from hypotension. Physiotherapy session is resumed at a later time or the next day when $\mathrm{PH}$ or symptoms resolves and is continued daily in ward until discharge.

$\mathrm{PH}$ is defined as either a drop of more than or equal to $20 \mathrm{~mm} \mathrm{Hg}$ in systolic blood pressure, or a drop of more than or equal to $10 \mathrm{~mm} \mathrm{Hg}$ in diastolic blood pressure during standing compared with supine position.

\section{Statistical Analysis}

Patient demographics and baseline characteristics, as well as perioperative factors, were analyzed. Categorical data were presented using number and percentages. Associations between categorical variables were tested using the Pearson's Chi-square test or Fisher's exact test if at least one of the expected cell counts was below 5. Continuous data were assessed if it was normally distributed through histograms and measures of kurtosis. Mean and standard deviation (SD) were presented for normally distributed data; and median 
Table 1 Baseline characteristics of patients who received dexamethasone versus patients who did not

\begin{tabular}{|c|c|c|c|c|}
\hline \multirow[t]{2}{*}{ Characteristics } & \multirow{2}{*}{$\begin{array}{l}\text { Overall }(n=149) \\
n(\%)\end{array}$} & Dexamethasone received $(n=78)$ & No dexamethasone $(n=71)$ & \multirow[t]{2}{*}{$p$-Value } \\
\hline & & $n(\%)$ & $n(\%)$ & \\
\hline $\begin{array}{l}\text { Age } \\
\text { Median (IQR) }\end{array}$ & $68(63,74)$ & $68(63,72)$ & $70(64,76)$ & 0.052 \\
\hline \multicolumn{4}{|l|}{ Gender } & \multirow[t]{3}{*}{0.90} \\
\hline Male & 49 (32.9) & $26(33.3)$ & $23(32.4)$ & \\
\hline Female & $100(67.1)$ & $52(66.7)$ & $48(67.6)$ & \\
\hline \multicolumn{5}{|l|}{ Comorbidities } \\
\hline Diabetes & $39(26.2)$ & $22(28.2)$ & $17(23.9)$ & 0.56 \\
\hline Hypertension & $104(69.8)$ & $57(73.1)$ & $47(66.2)$ & 0.36 \\
\hline Ischemic heart disease & $24(16.1)$ & $14(17.9)$ & $10(14.1)$ & 0.52 \\
\hline \multicolumn{4}{|l|}{ IV drip } & \multirow[t]{3}{*}{0.27} \\
\hline No & $48(32.2)$ & $22(28.2)$ & $26(36.6)$ & \\
\hline Yes & $101(67.8)$ & $56(71.8)$ & $45(63.4)$ & \\
\hline \multicolumn{4}{|l|}{ Anesthesia type } & \multirow[t]{3}{*}{0.45} \\
\hline Spinal & $94(63.1)$ & $47(60.3)$ & $47(66.2)$ & \\
\hline General anesthesia & $55(36.9)$ & $31(39.7)$ & $24(33.8)$ & \\
\hline \multicolumn{4}{|l|}{$\begin{array}{l}\text { Hb difference } \\
\text { Median (IQR) }\end{array}$} & \multirow[t]{2}{*}{0.64} \\
\hline Post-op-pre-op & $-1.2(-1.7,-0.7)$ & $-1.3(-1.8,-0.7)$ & $-1.2(-1.7,-0.8)$ & \\
\hline Net fluid balance & $500(400,700)$ & $500(400,650)$ & $600(450,700)$ & 0.33 \\
\hline
\end{tabular}

Abbreviations: Hb, hemoglobin; IQR, interquartile range; IV, intravenous; post-op, postoperative; Pre-op, preoperative.

and interquartile range (IQR) were presented for skewed data. Tests of association of continuous data between two groups were performed using the Student's $t$-test (if normally distributed), or the Wilcoxon's rank-sum test (if skewed). Statistical significance was declared if a two-tailed $p$-value was less than 0.05 .

Binary logistic regression was used to assess the effect of the use of dexamethasone on PH. The crude odds ratio (OR), together with the $95 \%$ confidence interval $(\mathrm{CI})$ and $p$-value, was computed to show the impact of dexamethasone on PH. The adjusted OR, after accounting for potential confounders such as age, comorbidities, and difference in hemoglobin level (pre- and postoperation), was also computed. Binary logistic regression was also used in the assessment of the use of dexamethasone on secondary outcomes, such as PONV, as well as whether patient was able to participate in early physiotherapy.

All analyses were done using STATA 16.1. (Reference: StataCorp. 19. Stata Statistical Software: Release 16. College Station, TX: StataCorp LLC.)

\section{Results}

\section{Patient Characteristics}

The baseline characteristics between patients who received dexamethasone and patients who did not receive dexamethasone were similar ( - Table $\mathbf{1}$ ).

\section{Outcomes between Patients who Received Dexamethasone and Those Who Did Not}

The effect of dexamethasone use on all three outcomes, that is, $\mathrm{PH}, \mathrm{PONV}$, and patient's ability to participate in POD 0/1 physiotherapy, was statistically significant (-Table 2 ).

Patients who received dexamethasone seemed to be less likely to have PH and PONV and were more likely to be able to participate in POD 0/1 physiotherapy. Also, 9.0\% of patients who used dexamethasone had $\mathrm{PH}$, as compared with $25.4 \%$ of patients who did not receive dexamethasone. Again, $7.7 \%$ of patients who received dexamethasone had PONV, as compared with $26.8 \%$ of patients who did not. Also, $93.6 \%$ of patients who received dexamethasone were able to walk and participate in physiotherapy, as compared with $83.1 \%$ of patients who did not.

After accounting for all potential confounders, patients who received dexamethasone were less likely to suffer from $\mathrm{PH}$. The adjusted odds of PH among patients who did not receive dexamethasone was 3.45 (95\% CI: 1.33-9.09; $p=0.03$ ) times as likely as the odds among patients who received dexamethasone ( - Table 3 ).

After accounting for all potential confounders, patients who received dexamethasone were less likely to develop PONV. The adjusted odds of PONV among patients who did not receive dexamethasone was 4.76 (95\% CI: 1.56-14.3; $p=0.006$ ) times as likely as the odds among patients who received dexamethasone ( - Table 4 ). 
Table 2 Comparison of outcomes between patients who received dexamethasone and patients who did not

\begin{tabular}{|c|c|c|c|c|}
\hline \multirow[t]{2}{*}{ Outcomes } & Overall $(n=149)$ & $\begin{array}{l}\text { Dexamethasone } \\
\text { received }(n=78)\end{array}$ & $\begin{array}{l}\text { No dexamethasone } \\
(n=71)\end{array}$ & \multirow[t]{2}{*}{$p$-Value } \\
\hline & $n(\%)$ & $n(\%)$ & $n(\%)$ & \\
\hline \multicolumn{4}{|l|}{$\mathrm{PH}$} & \multirow[t]{3}{*}{0.008} \\
\hline No & 124 (83.2) & $71(91.0)$ & $53(74.7)$ & \\
\hline Yes & $25(16.8)$ & $7(9.0)$ & $18(25.4)$ & \\
\hline \multicolumn{4}{|l|}{ Any PONV? } & \multirow[t]{3}{*}{0.002} \\
\hline No & $124(83.2)$ & $72(92.3)$ & $52(73.2)$ & \\
\hline Yes & $25(16.8)$ & $6(7.7)$ & $19(26.8)$ & \\
\hline \multicolumn{4}{|l|}{$\begin{array}{l}\text { Was patient able to walk or } \\
\text { participate in physiotherapy? }\end{array}$} & \multirow[t]{3}{*}{0.04} \\
\hline No & 17 (11.4) & $5(6.4)$ & $12(16.9)$ & \\
\hline Yes & $132(88.6)$ & 73 (93.6) & $59(83.1)$ & \\
\hline
\end{tabular}

Abbreviations: $\mathrm{PH}$, postural hypotension; PONV, postoperative nausea and vomiting.

After accounting for all potential confounders, patients who received dexamethasone were more likely to be able to participate in POD 0/1 physiotherapy. The adjusted odds of patient's ability to participate in POD 0/1 physiotherapy among patients who received dexamethasone was 2.42 (95\% CI: $0.74-7.86 ; p=0.14$ ) times as likely as the odds among patients who did not receive dexamethasone. However, this was not statistically significant, $p=0.14$ (-Table 5).

Interestingly, it was observed that there was a statistically significant dose-dependent relationship between dexamethasone and $\mathrm{PH}(p=0.03)$. Patients who received $12 \mathrm{mg}$ ( $4 \mathrm{mg}$ at 8-hour regime) of dexamethasone were 0.25 times (95\% CI: 0.09-0.73) as likely to suffer from PH, as compared with patients who did not receive any dexamethasone while patients who received $24 \mathrm{mg}$ ( $8 \mathrm{mg}$ at 8 -hour regime) of dexamethasone were 0.45 times (95\% CI: $0.09-2.20$ ) as likely to suffer from $\mathrm{PH}$, as compared with patients who did not receive any dexamethasone ( - Table $\mathbf{6}$ ).

A statistically significant dose-dependent relationship was also observed between dexamethasone dose and PONV ( $p=0.009$ ). Patients who received $12 \mathrm{mg}$ of dexamethasone were 0.14 times (95\% CI: 0.04-0.49) as likely to suffer from PONV, as compared with patients who did not receive any dexamethasone. Patients who received $24 \mathrm{mg}$ of dexamethasone were 0.68 times (95\% CI: $0.17-2.69$ ) as likely to suffer from PONV, as compared with patients who did not

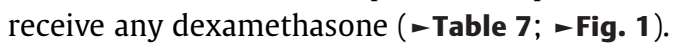

\section{Discussion}

ERAS protocols have been implemented internationally for high-volume orthopaedic procedures, notably for TKR and total hip replacement (THR) surgeries, following its track record in reducing hospital length of stay and complications. ${ }^{1}$ In the era of the novel coronavirus disease 2019 (COVID-19) pandemic where health care resources have been diverted to attend to the crisis, this is especially relevant to increase efficiency of hospital resource utiliza- tion. ${ }^{6}$ The key to fast-track postoperative recovery is achieving early postoperative mobilization through optimal pain and blood loss control, and prevention of PH and PONV. In this paper, we want to focus on PH and PONV and the effects of dexamethasone on postoperative early mobilization. This has been studied frequently in THR patients, with 19.2 to $46.2 \%$ prevalence rates of $\mathrm{PH}^{8}{ }^{8,9}$ However, plausible literature exists for PH in TKR patients. Zhang et al quoted a 12.3\% prevalence rate of $\mathrm{PH}$ in 495 patients who underwent TKR for primary osteoarthritis. ${ }^{10}$ This is comparable with our prevalence rate of $16.8 \%$. PH can present clinically as orthostatic intolerance through a constellation of symptoms including dizziness, nausea, vomiting, and visual disturbances. ${ }^{11,12}$ The pathophysiology of postoperative PH is likely multifactorial and changes in cerebral oxygenation, cardiovascular regulation, and autonomic dysfunction have been recognized as significant mechanisms. ${ }^{8,9,13}$ A prospective study by Jans et al found that orthostatic intolerance was associated with decreased cardiac output and systemic vascular resistance upon standing and cerebral hypoperfusion. ${ }^{22}$ Surgical insult causes a systematic inflammatory response which stimulates the vagus nerve, producing an "inflammatory reflex."2 This is a cholinergic anti-inflammatory pathway which could produce vasodilation, bradycardia, hence downregulating and dysregulating the blood pressure. The type and extent of surgical trauma is likely to affect the magnitude of inflammatory response as well. This may explain the lower prevalence of PH in TKR compared with THR, as TKR tends to have less blood loss and shorter operating time.

Dexamethasone is a synthetic glucocorticoid that is widely used as an anti-inflammatory agent in autoimmune and inflammatory disorders. Its regulation of the hypothalamicpituitary-adrenal axis downregulates the release of inflammatory cytokines like bradykinin, interleukin (IL)-1, IL-2, and IL-6. ${ }^{5}$ It is a safe perioperative agent in TKR surgery with good analgesic and antiemetic actions without influencing woundrelated complication rates. ${ }^{14-16}$ Interestingly, its mechanism of action as an antiemetic agent is unknown, with various 
Table 3 Binary logistic regression analysis on $\mathrm{PH}$

\begin{tabular}{|c|c|c|c|c|c|c|}
\hline & \multicolumn{3}{|c|}{ Unadjusted } & \multicolumn{3}{|l|}{ Adjusted } \\
\hline & Crude OR & $95 \% \mathrm{Cl}$ & $p$-Value & Adjusted OR & $95 \% \mathrm{Cl}$ & $p$-Value \\
\hline \multicolumn{6}{|l|}{ Dexamethasone } & \multirow[t]{3}{*}{0.03} \\
\hline No & 1 (ref.) & & & 1 (ref.) & & \\
\hline Yes & 0.29 & $0.11,0.75$ & 0.01 & 0.31 & $0.11,0.90$ & \\
\hline Age & 1.04 & $0.98,1.10$ & 0.22 & 1.03 & $0.97,1.10$ & 0.30 \\
\hline \multicolumn{6}{|l|}{ Gender } & \multirow[t]{3}{*}{0.58} \\
\hline Male & 1 (ref.) & & & 1 (ref.) & & \\
\hline Female & 0.85 & $0.34,2.08$ & 0.72 & 1.36 & $0.45,4.08$ & \\
\hline \multicolumn{6}{|l|}{ Diabetes mellitus } & \multirow[t]{3}{*}{0.06} \\
\hline No & 1 (ref.) & & & 1 (ref.) & & \\
\hline Yes & 0.33 & $0.09,1.18$ & 0.09 & 0.20 & $0.04,1.06$ & \\
\hline \multicolumn{6}{|l|}{ Hypertension } & \multirow[t]{3}{*}{0.42} \\
\hline No & 1 (ref.) & & & 1 (ref.) & & \\
\hline Yes & 0.73 & $0.29,1.80$ & 0.49 & 0.63 & $0.21,1.92$ & \\
\hline \multicolumn{6}{|l|}{ Ischemic heart disease } & \multirow[t]{3}{*}{0.02} \\
\hline No & 1 (ref.) & & & 1 (ref.) & & \\
\hline Yes & 1.86 & $0.65,5.29$ & 0.25 & 5.03 & $1.33,19.06$ & \\
\hline \multicolumn{6}{|l|}{ IV drip } & \multirow[t]{3}{*}{0.14} \\
\hline No & 1 (ref.) & & & 1 (ref.) & & \\
\hline Yes & 0.66 & $0.27,1.61$ & 0.36 & 0.45 & $0.15,1.30$ & \\
\hline \multicolumn{6}{|l|}{ Anesthesia type } & \multirow[t]{3}{*}{0.27} \\
\hline Spinal & 1 (ref.) & & & 1 (ref.) & & \\
\hline General anesthesia & 0.62 & $0.24,1.58$ & 0.31 & 0.54 & $0.18,1.61$ & \\
\hline \multicolumn{7}{|l|}{ Hb difference } \\
\hline Post-op-pre-op & 1.45 & $0.88,2.37$ & 0.14 & 1.49 & $0.82,2.71$ & 0.19 \\
\hline Net fluid balance & 1.001 & $0.99,1.002$ & 0.15 & 1.001 & $1.0001,1.003$ & 0.04 \\
\hline
\end{tabular}

Abbreviations: $\mathrm{Cl}$, confidence interval; $\mathrm{Hb}$, hemoglobin; IV, intravenous; OR, odds ratio; PH, postural hypotension; post-op, postoperative; Pre-op, preoperative; ref. reference.

Table 4 Binary logistic regression analysis on PONV

\begin{tabular}{|l|l|l|l|l|l|l|}
\hline & \multicolumn{2}{|l|}{ Unadjusted } & \multicolumn{2}{l|}{ Adjusted } \\
\hline & Crude OR & $95 \% \mathrm{Cl}$ & $p$-Value & \multicolumn{1}{l|}{ Adjusted ${ }^{\text {a }}$ OR } & $95 \% \mathrm{Cl}$ & $p$-Value \\
\hline Dexamethasone & & & & & 0.006 \\
\hline Yes & 1 (ref.) & & & 1 (ref.) & & \\
\hline No & 4.35 & $1.64,11.1$ & 0.003 & 4.76 & $1.56,14.3$ \\
\hline
\end{tabular}

Abbreviations: $\mathrm{Cl}$, confidence interval; OR, odds ratio; PONV, postoperative nausea and vomiting; ref. reference.

${ }^{a}$ Adjusted for age, gender, diabetes mellitus, hypertension, ischemic heart disease, intravenous drip, anesthesia type, difference in hemoglobin level, and net balance.

postulations including the depletion of gamma aminobutyric acid (GABA) stores, reduction of blood-brain barrier to emetogenic toxins, inhibition of central prostaglandins, and serotonin. ${ }^{17}$ Because of its versatility, safety, and ready availability, this prompted the operating surgeon of this study to introduce it into the postoperative medication regimen.

In our study, patients who received postoperative intravenous dexamethasone over 24 hours were less likely to develop $\mathrm{PH}$, adjusted $\mathrm{OR}=0.31 \quad$ (95\% CI: 0.11-0.90), $p=0.03$, and were also less likely to develop PONV, adjusted $\mathrm{OR}=0.21(95 \% \mathrm{CI}: 0.07-0.64), p=0.006$, compared with those who did not receive it. This was based on a regimen of an 8-hour dosing of intravenous dexamethasone over 24 hours, with most of the patients $(80.8 \%)$ receiving a cumulative dose of $12 \mathrm{mg}$, and the remaining (19.2\%) $24 \mathrm{mg}$. This is the first study evaluating the dose dependent relationship of dexamethasone on $\mathrm{PH}$ and PONV. In a metaanalysis of randomized controlled trials, de Oliveira et al 
Table 5 Binary logistic regression analysis on patient's ability to participate in POD 0/1 physiotherapy

\begin{tabular}{|c|c|c|c|c|c|c|}
\hline & \multicolumn{3}{|c|}{ Unadjusted } & \multicolumn{3}{|l|}{ Adjusted } \\
\hline & Crude OR & $95 \% \mathrm{Cl}$ & $p$-Value & Adjusted $^{\mathrm{a}} \mathrm{OR}$ & $95 \% \mathrm{Cl}$ & $p$-Value \\
\hline Dexamethasone & & & & & & 0.14 \\
\hline No & 1 (ref.) & & & 1 (ref.) & & \\
\hline Yes & 2.97 & $0.99,8.91$ & 0.052 & 2.42 & $0.74,7.86$ & \\
\hline
\end{tabular}

Abbreviations: $\mathrm{Cl}$, confidence interval; OR, odds ratio; POD, postoperative day; ref. reference.

${ }^{a}$ Adjusted for age, gender, diabetes mellitus, hypertension, ischemic heart disease, intravenous drip, anesthesia type, difference in hemoglobin level, and net fluid balance.

Table 6 Crude OR of dexamethasone dose on $\mathrm{PH}$

\begin{tabular}{|l|l|l|l|l|}
\hline & No. (\%) & Crude OR & $95 \% \mathrm{Cl}$ & $p$-Value \\
\hline Dexamethasone dose & & & & 0.03 \\
\hline Nil & 71 & 1 (ref.) & & \\
\hline $12 \mathrm{mg}$ & 63 & 0.25 & $0.09,0.73$ & 0.01 \\
\hline $24 \mathrm{mg}$ & 15 & 0.45 & $0.09,2.20$ & 0.33 \\
\hline
\end{tabular}

Abbreviations: $\mathrm{Cl}$, confidence interval; OR, odds ratio; $\mathrm{PH}$, postural hypotension; ref. reference.

Table 7 Crude OR of dexamethasone dose on PONV

\begin{tabular}{|l|l|l|l|l|}
\hline & $\begin{array}{l}\text { No. } \\
(\%)\end{array}$ & $\begin{array}{l}\text { Crude } \\
\text { OR }\end{array}$ & $95 \% \mathrm{Cl}$ & $p$-Value \\
\hline $\begin{array}{l}\text { Dexamethasone } \\
\text { dose }\end{array}$ & & & & 0.009 \\
\hline Nil & 71 & 1 (ref.) & & \\
\hline $12 \mathrm{mg}$ & 63 & 0.14 & $0.04,0.49$ & 0.002 \\
\hline $24 \mathrm{mg}$ & 15 & 0.68 & $0.17,2.69$ & 0.59 \\
\hline
\end{tabular}

Abbreviations: $\mathrm{Cl}$, confidence interval; OR, odds ratio; PONV, postoperative nausea and vomiting; ref. reference.

studied perioperative single dexamethasone dosing in three groups; low $(<0.1 \mathrm{mg} / \mathrm{kg})$, intermediate $(0.11-0.2 \mathrm{mg} / \mathrm{kg})$, and high $(>0.21 \mathrm{mg} / \mathrm{kg})$. They found that a dosing of $>0.1$ $\mathrm{mg} / \mathrm{kg}$ is an effective adjunct in multimodal strategies to reduce postoperative pain and opioid consumption after surgery. ${ }^{18}$ Another meta-analysis by Waldron et al divided studies with single dexamethasone dosing into 4 to $5 \mathrm{mg}$ and 8 to $10 \mathrm{mg}$ groups, but did not find dose-dependent effects on its analgesic effect. ${ }^{19}$ Similarly, our study found that a higher dexamethasone dosing $(24 \mathrm{mg})$ was not significantly associated with lower PH nor PONV rates, compared with a lower dosing $(12 \mathrm{mg})$ and therefore the authors have adopted the use of 12-mg dosing. However, a randomized controlled trial by Lunn et al studied 48 patients who underwent unilateral TKR surgery and found that a single preoperative high dose of intravenous methylprednisolone did not have a sustained analgesic effect, ${ }^{20}$ and hence suggested possible value in prolonged steroid administration.

Despite the use of dexamethasone being associated with lower rates of $\mathrm{PH}$ and PONV in our study, there was no significant difference in the outcome of early mobilization, adjusted $\mathrm{OR}=2.42$ (95\% CI: 0.74-7.86), $p=0.14$. In an Australian prospective study of 989 patients who underwent TKR, Chua et al evaluated patient- and treatment-related factors. $^{21}$ Treatment-related factors, namely, hospital site and absence of blood transfusion, were associated with postoperative day of mobilization, on multivariate analysis. Other treatment-related factors include use of regional anesthesia which may cause motor blockade, insertion of indwelling urinary catheter, and even acute postoperative complications, such as the aforementioned orthostatic intolerance and PONV, though not significant. Similarly, our study did not find association between patient-related factors, that is, presence of existing hypertension or diabetes mellitus, and early mobilization. Furthermore, none of the treatmentrelated factors studied, that is, choice of anesthesia, use of intravenous drip, net fluid balance, and drop in hemoglobin count, were associated either. Our institution's TKR ERAS protocol is audited regularly for compliance, and the reinforcement of early mobilization is ensured by the multidisciplinary team comprising the orthopaedic-trained specialized nurses, the operating team, and musculoskeletal physiotherapists. In the study by Chua et al, the differences in mobilization protocols and the stringency of execution by service providers may have a downstream effect on the actual time of mobilization postoperatively. Multicenter studies may be beneficial to assess the impact of differences in hospital protocol on early mobilization and other outcomes.

The strengths of this study include consistency of the TKR surgical protocol and inpatient management. All TKRs were performed by the same adult reconstruction team comprising two specialist surgeons, with standardized surgical steps and perioperative setups, as well as postoperative protocols. Additionally, this is the first study evaluating postoperative $\mathrm{PH}$ in TKRs and the dose-dependent relationship of dexamethasone on PH and PONV. Other studies have looked at methods to reduce PH in THR surgery and in other specialty surgery, through the use of intravenous methylprednisolone and oral midodrine hydrochloride. ${ }^{9,22}$ 


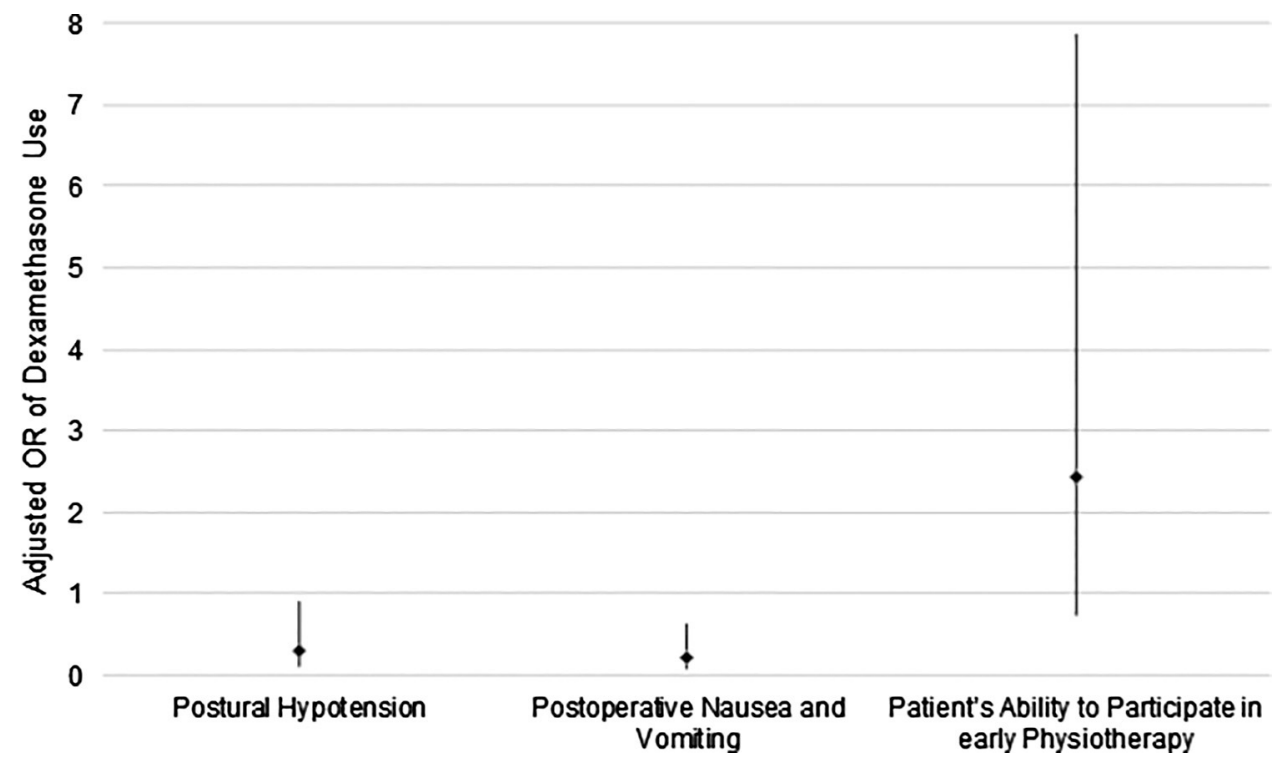

Fig. 1 Adjusted OR of dexamethasone use on outcomes. OR has been adjusted for age, gender, diabetes mellitus, hypertension, ischemic heart disease, IV drip, anesthesia type, difference in hemoglobin level, and net fluid balance. Lines denote lower $95 \% \mathrm{Cl}$ and upper $95 \% \mathrm{Cl}$ of the adjusted OR. Cl, confidence interval; OR, odds ratio.

\section{Limitations}

Besides the inherent bias of a retrospective study, the limitations of our study are that we did not assess the effects of dexamethasone administration on blood glucose levels of the patients which would allow us to better assess the safe and optimal dosing range. The key concerns of steroid use are hyperglycemia and wound infection, and this is relevant in our patient group with a diabetes mellitus prevalence of $26.2 \%$. However, the 12 to $24 \mathrm{mg}$ cumulative dosing is considered a moderate dosing, and large studies have shown that dexamethasone use was not associated with postoperative wound infection, even after adjusting for diabetes mellitus. ${ }^{23,24}$ None of the patients in our study had prosthetic joint infections. We also did not assess the postoperative analgesic effects of dexamethasone in our study population.

\section{Conclusion}

The clinical impact of this study is that it supports the routine use of postoperative dexamethasone, as it is significantly associated with lower rates of postoperative $\mathrm{PH}$ and PONV after TKR. In addition, a lower dose of dexamethasone dosing of $12 \mathrm{mg}$ over 24 hours is sufficient to achieve adequate PONV and $\mathrm{PH}$ prophylaxis compared with a higher dosing of $24 \mathrm{mg}$ over 24 hours.

\section{Conflict of Interest}

None declared.

\section{Acknowledgment}

The authors would like to thank Ms Lim Gek Hsiang and Ms Cheng Jia Min Julia for their guidance in the statistical analysis of this study.

\section{References}

1 Wainwright TW, Gill M, McDonald DA, et al. Consensus statement for perioperative care in total hip replacement and total knee replacement surgery: Enhanced Recovery After Surgery (ERAS) Society recommendations. Acta Orthop 2020;91(01): 3-19

2 Huston JM, Tracey KJ. The pulse of inflammation: heart rate variability, the cholinergic anti-inflammatory pathway and implications for therapy. J Intern Med 2011;269(01):45-53

3 Jans $\emptyset$, Brinth L, Kehlet $H$, Mehlsen J. Decreased heart rate variability responses during early postoperative mobilizationan observational study. BMC Anesthesiol 2015;15:120

4 Weibel S, Rücker G, Eberhart LH, et al. Drugs for preventing postoperative nausea and vomiting in adults after general anaesthesia: a network meta-analysis. Cochrane Database Syst Rev 2020;10:CD012859

5 Rhen T, Cidlowski JA. Antiinflammatory action of glucocorticoids-new mechanisms for old drugs. N Engl J Med 2005;353 (16):1711-1723

6 Ding BTK, Ng J, Tan KG. Enhanced recovery after surgery for knee arthroplasty in the era of COVID-19. J Knee Surg 2020 (e-pub ahead of print). Doi: 10.1055/s-0040-1715125

7 Amirshahi M, Behnamfar N, Badakhsh M, et al. Prevalence of postoperative nausea and vomiting: a systematic review and meta-analysis. Saudi J Anaesth 2020;14(01):48-56

8 Jans $\emptyset$, Bundgaard-Nielsen M, Solgaard S, Johansson PI, Kehlet H. Orthostatic intolerance during early mobilization after fast-track hip arthroplasty. Br J Anaesth 2012;108(03):436-443

9 Lindberg-Larsen V, Petersen PB, Jans Ø, Beck T, Kehlet H. Effect of pre-operative methylprednisolone on orthostatic hypotension during early mobilization after total hip arthroplasty. Acta Anaesthesiol Scand 2018;62(07):882-892

10 Zhang Y-M, He J, Zhou C, Li Y, Yi D-K, Zhang X. Acute hypotension after total knee arthroplasty and its nursing strategy. Int J Clin Exp Med 2015;8(08):13946-13953

11 Grubb BP. Neurocardiogenic syncope and related disorders of orthostatic intolerance. Circulation 2005;111(22):2997-3006

12 Cowie DA, Shoemaker JK, Gelb AW. Orthostatic hypotension occurs frequently in the first hour after anesthesia. Anesth Analg 2004;98(01):40-45 
13 Jans $\emptyset$, Kehlet H. Postoperative orthostatic intolerance: a common perioperative problem with few available solutions. Can J Anaesth 2017;64(01):10-15

14 Samona J, Cook C, Krupa K, et al. Effect of intraoperative dexamethasone on pain scores and narcotic consumption in patients undergoing total knee arthroplasty. Orthop Surg 2017;9(01): $110-114$

15 Li X, Xu G, Xie W, Ma S. The efficacy and safety of dexamethasone for pain management after total knee arthroplasty: a systematic review and meta-analysis. Int J Surg 2018;53:65-71

16 DREAMS Trial Collaborators and West Midlands Research Collaborative. Dexamethasone versus standard treatment for postoperative nausea and vomiting in gastrointestinal surgery: randomised controlled trial (DREAMS Trial). BMJ 2017;357: j1455. Doi: 10.1136/bmj.j1455

17 Gupta B. Role of dexamethasone in peri-operative anesthesia management: a review of literature. Res Practice Anesth 2017;2 (02):33-39

18 De Oliveira GS Jr., Almeida MD, Benzon HT, McCarthy RJ. Perioperative single dose systemic dexamethasone for postoperative pain: a meta-analysis of randomized controlled trials. Anesthesiology 2011;115(03):575-588

19 Waldron NH, Jones CA, Gan TJ, Allen TK, Habib AS. Impact of perioperative dexamethasone on postoperative analgesia and side-effects: systematic review and meta-analysis. $\mathrm{Br} \mathrm{J}$ Anaesth 2013;110(02):191-200

20 Lunn TH, Kristensen BB, Andersen LØ, et al. Effect of high-dose preoperative methylprednisolone on pain and recovery after total knee arthroplasty: a randomized, placebo-controlled trial. $\mathrm{Br} \mathrm{J}$ Anaesth 2011;106(02):230-238

21 Chua MJ, Hart AJ, Mittal R, Harris IA, Xuan W, Naylor JM. Early mobilisation after total hip or knee arthroplasty: A multicentre prospective observational study. PLoS One 2017;12(06):e0179820

22 Jans Ø, Mehlsen J, Kjærsgaard-Andersen P, et al. Oral midodrine hydrochloride for prevention of orthostatic hypotension during early mobilization after hip arthroplasty: a randomized, doubleblind, placebo-controlled trial. Anesthesiology 2015;123(06): 1292-1300

23 Richardson AB, Bala A, Wellman SS, Attarian DE, Bolognesi MP, Grant SA. Perioperative dexamethasone administration does not increase the incidence of postoperative infection in total hip and knee arthroplasty: a retrospective analysis. J Arthroplasty 2016; 31(08):1784-1787

24 Corcoran T, Kasza J, Short TG, et al; ENIGMA-II investigators. Intraoperative dexamethasone does not increase the risk of postoperative wound infection: a propensity score-matched post hoc analysis of the ENIGMA-II trial (EnDEX). Br J Anaesth 2017;118(02):190-199 